\title{
Capsule Migration and Deformation in a Converging Micro-Capillary
}

\author{
Yiyang Wang and Panagiotis Dimitrakopoulos * \\ Department of Chemical and Biomolecular Engineering, University of Maryland, College Park, MD 20742, USA; \\ yiyang91@email.arizona.edu \\ * Correspondence: dimitrak@umd.edu
}

check for updates

Citation: Wang, Y.; Dimitrakopoulos, P. Capsule Migration and Deformation in a Converging Micro-Capillary. Processes 2021, 9 , 452. https://doi.org/10.3390/ pr9030452

Academic Editor: Md. Shakhaoath Khan

Received: 3 February 2021

Accepted: 25 February 2021

Published: 3 March 2021

Publisher's Note: MDPI stays neutral with regard to jurisdictional claims in published maps and institutional affiliations.

Copyright: (c) 2021 by the authors. Licensee MDPI, Basel, Switzerland. This article is an open access article distributed under the terms and conditions of the Creative Commons Attribution (CC BY) license (https:/ / creativecommons.org/licenses/by/ $4.0 /)$.
Abstract: The lateral migration of elastic capsules towards a microchannel centerline plays a major role in industrial and physiological processes. Via our computational investigation, we show that a constriction connecting two straight microchannels facilitates the lateral capsule migration considerably, which is relatively slow in straight channels. Our work reveals that the significant cross-streamline migration inside the constriction is dominated by the strong hydrodynamic forces due to the capsule size. However, in the downstream straight channel, the increased interfacial deformation at higher capillary numbers or a lower viscosity ratio and lower membrane hardness results in increased lateral cross-streamline migration. Thus, our work highlights the different migration mechanisms occurring over curved and straight streamlines.

Keywords: elastic capsule; microfluidics; stokes flow

\section{Introduction}

The study of the interfacial dynamics of artificial or physiological capsules (i.e., membrane-enclosed fluid volumes) in viscous flows has seen increased interest during the last few decades, owing to their numerous engineering and biomedical applications [1,2]. In the area of interest of the present work, the flow-induced lateral migration of soft particles (such as droplets, elastic capsules, vesicles or erythrocytes) plays a significant role in industrial and physiological processes [3-6]. Common examples include the erythrocyte migration in blood vessels and the migration of soft particles towards a device centerline which is desirable for the targeted drag delivery and for fabrication or characterization purposes [7-10].

Owing to the small particle size and the microdevice flow properties, lateral migration often happens under inertialess conditions, provided that the symmetry of the Stokes flow is broken owing to the particle deformation, which may occur due to the shear flow close to a solid boundary or the shear-gradient of the Poiseuille flow in a channel [3,4]. However, in straight channels (i.e., straight streamlines), the lateral migration is quite slow $[9,11]$. Typically, the migration velocity is $O\left(10^{-2}\right)$ or even less of the channel's main velocity, and thus a long channel is required to achieve considerable lateral migration [11,12]. (Note that for a migration distance comparable to the particle size, a microchannel with a length higher than 100 times the particle size is required [11,13]).

To facilitate the cross-streamline migration towards the channel centerline, in this work we propose flowing elastic capsules in a converging micro-capillary of comparable size under Stokes flow conditions. Our work highlights the different migration mechanisms occurring over curved and straight streamlines. In particular, our investigation shows that the significant cross-streamline migration over the curved streamlines inside the constriction is dominated by the strong hydrodynamic forces due to the capsule size. However, the interfacial deformation does facilitate the lateral migration over the straight streamlines of the downstream straight channel, as our results for varying capillary number, viscosity ratio and membrane hardness reveal. 


\section{Problem Description}

In this work, we investigate the motion and deformation of an elastic capsule flowing through a straight micro-channel made of two square channels with a converging section in the middle, as shown in Figure 1. The three-dimensional capsule consists of a fluid volume enclosed by a thin elastic membrane with a spherical, undisturbed shape. For illustration purposes, we assume that the channel is horizontal, as shown in Figure 1a. Thus, the flow direction (i.e., the $x$-axis) will be referred to as length, while the $z$ and $y$-directions will be referred as height and width, respectively. The height of the upstream square channel is $3 \ell$, while that of the downstream square channel is $2 \ell$. The converging middle section, connecting the two square channels, has length $\ell_{\text {con }}=\ell$. The half-height $\ell$ of the downstream square channel serves as the length scale for this problem, while the origin of the coordinate system is placed at the beginning of this channel (just after the converging section), as illustrated in Figure 1a.

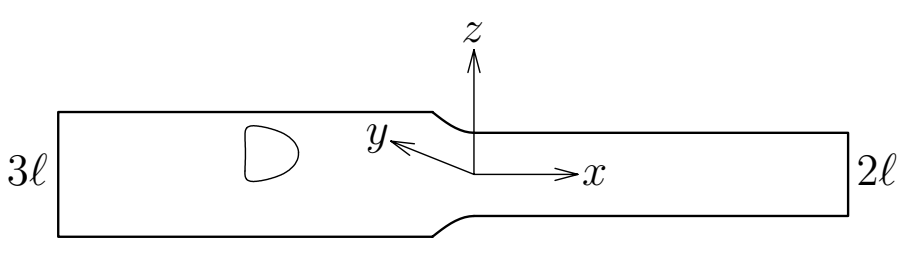

(a)

(b)

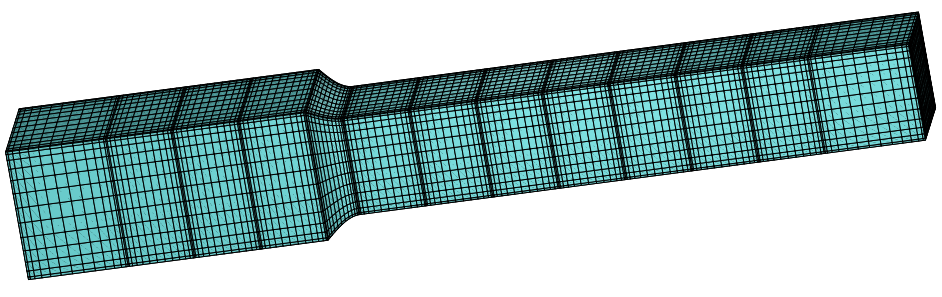

Figure 1. (a) Illustration of an elastic capsule flowing off the centerline of a converging square micro-channel. (b) Spectral boundary element discretization of the micro-capillary geometry.

The shape of the converging middle section in our micro-geometry is defined via a "quarter-cosine" variation, which for the coordinate system shown in Figure 1a is given by

$$
f(x)=\ell\left\{\frac{3}{2}+\frac{1}{2} \cos \left[\left(\frac{x}{\ell}+2\right) \frac{\pi}{2}\right]\right\}
$$

where $-1 \leq x / \ell \leq 0$ and $f(x)$ defines the geometry's height $z(x)$ or width $y(x)$. Similar micro-devices involving converging capillaries with circular or square cross-sections have been produced via glass fabrication to study the dynamics of soft particles, e.g., for the generation of monodisperse double emulsions and the elasticity determination of soft gels [14-16]. Note that we utilized the same micro-device to study centerline capsule motion in the constriction as a moduli determination method [17].

The capsule's interior and exterior are Newtonian fluids, with viscosities $\lambda \mu$ and $\mu$, and the same density. The capsule volume $V=4 \pi a^{3} / 3$ defines the capsule size $a$ which is smaller than the micro-device's half-height $\ell$. In our computations, the elastic capsule was $5 \%$ over-inflated, made of a thin strain-hardening membrane, following the Skalak et al. constitutive law [18] (and thus called Skalak capsule in this paper) with comparable shearing and area-dilatation resistance but negligible bending resistance. Thus, the membrane resistance is described by the shear and area-dilatation moduli $G_{S}$ and $G_{a}$, respectively. 
In particular, the in-plane tension tensor $\tau$ is described by the strain-hardening constitutive law of Skalak et al. [18] which relates $\tau^{\prime}$ s eigenvalues (or principal elastic tensions $\left.\tau_{\beta}^{P}, \beta=1,2\right)$ to the principal stretch ratios $\lambda_{\beta}$ by

$$
\tau_{1}^{P}=\frac{G_{s} \lambda_{1}}{\lambda_{2}}\left\{\lambda_{1}^{2}-1+C \lambda_{2}^{2}\left[\left(\lambda_{1} \lambda_{2}\right)^{2}-1\right]\right\}
$$

Note that the reference shape of the elastic tensions is the spherical quiescent shape of the capsule, and to calculate $\tau_{2}^{P}$, reverse the $\lambda_{\beta}$ subscripts [18,19].

This modeling represents artificial and biological capsules with thin membranes where the membrane tensions increase superlinearly with the applied strain $[19,20]$. The strength of the membrane's strain-hardening nature is described by the dimensionless membrane hardness $C$, which is associated with the scaled area-dilatation modulus $G_{a}$ of the membrane, $G_{a} / G_{s}=1+2 C[19]$.

At time $t=0$ the capsule is located off the centerline in the upstream square channel so that its centroid is $x_{c}=\left(x_{c}, y_{c}, z_{c}\right)=\left(-4 \ell, 0, z_{c}^{0}\right)$; the flow is turned on inside the microdevice; and we investigated the lateral migration of the capsule towards the device's centerline. We emphasize that the specific choice for the capsule's initial position $x_{c}$ does not affect the capsule dynamics inside the constriction or downstream of it, i.e., we obtained identical results even for capsules placed further upstream of the constriction. At the upstream and downstream ends of the micro-device far from the capsule, the flow approaches the single-phase Poiseuille flow in a square channel that serves as the boundary condition, while the flow rate $Q$ is fixed inside the micro-device. We assume that the Reynolds number is small for both the surrounding and the inner flows, and thus the capsule deformation occurs in the Stokes regime.

The present problem depends on the capsule's size $a / \ell$ and initial position $z_{c}^{0} / \ell$, the fluids viscosity ratio $\lambda$, the membrane's hardness $C$ and the capillary number $\mathrm{Ca}=\mu \mathcal{U} / G_{s}$ where $\mathcal{U}$ is the average undisturbed velocity at the downstream square micro-channel. Note that we investigated capsules with size $a / \ell=0.1-0.8$ and capillary number $\mathrm{Ca}=0.02-0.1$; these conditions can readily be used in experimental microfluidic systems; e.g., see [10,21]. The numerical solution of the interfacial Stokes flow problem was achieved through our membrane spectral boundary element method $[17,20]$. Convergence runs covering the entire interfacial evolution revealed that our results are accurate to at least two significant digits. The interested reader is referred to our recent publications for more details on our spectral boundary algorithm and our studies on capsule dynamics in microfluidic flows, e.g., [13,17,22-24]. An extended description of our capsule modeling and our computational method for centerline capsule motion in a constriction (used as a moduli determination method) has been included in the electronic supplementary information of [17].

\section{Results}

We begin our investigation by comparing the lateral migration of an elastic capsule inside two different microchannel geometries: a straight square channel and a constriction made of the same upstream square channel. The capsule of size $a / \ell=0.8$ is released at three different locations so that its initial centroid is $z_{c}^{0} / \ell=0.2,0.5$ or 0.8 , as shown in Figure 2 . The capsule has membrane hardness $C=1$, viscosity ratio $\lambda=1$ and capillary number $\mathrm{Ca}=0.1$.

As seen in Figure 2, all capsules migrate towards the channel centerline; however, the lateral migration in the straight channel is much slower than that in the constriction. This is due to the significant increase of the migration velocity $U_{z}$ inside the constriction which occupies the $x / \ell$-region $[-1,0]$, owing to the local strong hydrodynamic forces on the capsule surface. 

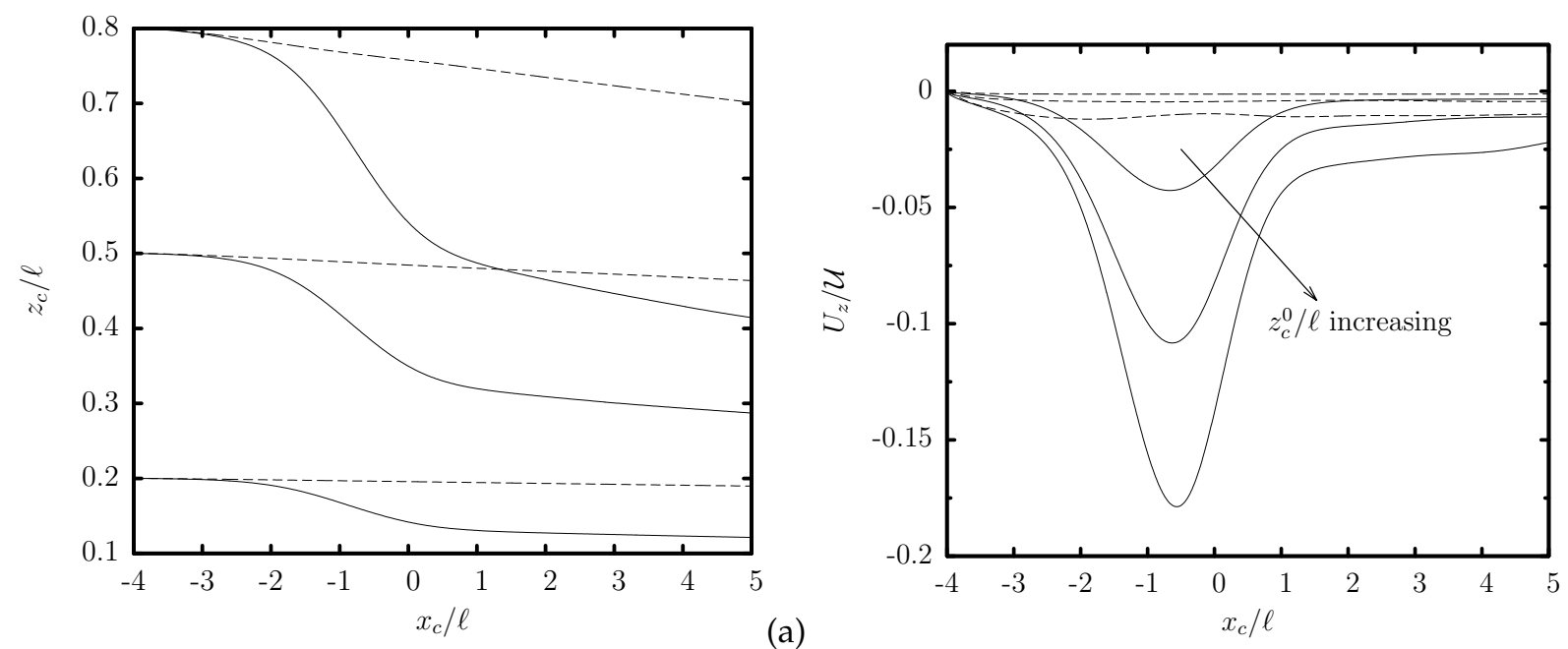

(b)

Figure 2. Capsule properties as a function of the centroid $x_{c}$, for a Skalak capsule with $a / \ell=0.8, \mathrm{Ca}=0.1, C=1, \lambda=1$ and initial position $z_{\mathcal{c}}^{0} / \ell=0.2,0.5,0.8$, moving inside a constriction (-) or a straight channel (---). (a) Capsule centroid $z_{c}$, and (b) migration velocity $U_{z}$.

Three-dimensional shapes of the capsule with initial position $z_{c}^{0} / l=0.8$ flowing through the constriction are shown in Figure 3. When the capsule moves inside the upstream square channel, the Poiseuille flow deforms and orients the capsule, producing a small lateral migration. As the capsule approaches the constriction, a higher deformation occurs and the capsule moves faster towards the device centerline. After the constriction, the capsule is more deformed in the smaller square channel on the right, owing to the higher average velocity while it is significantly closer to the device centerline. At this location, the capsule migration velocity is reduced compared to that inside the constriction, but is still higher than that in the straight channel, as shown in Figure 2b.
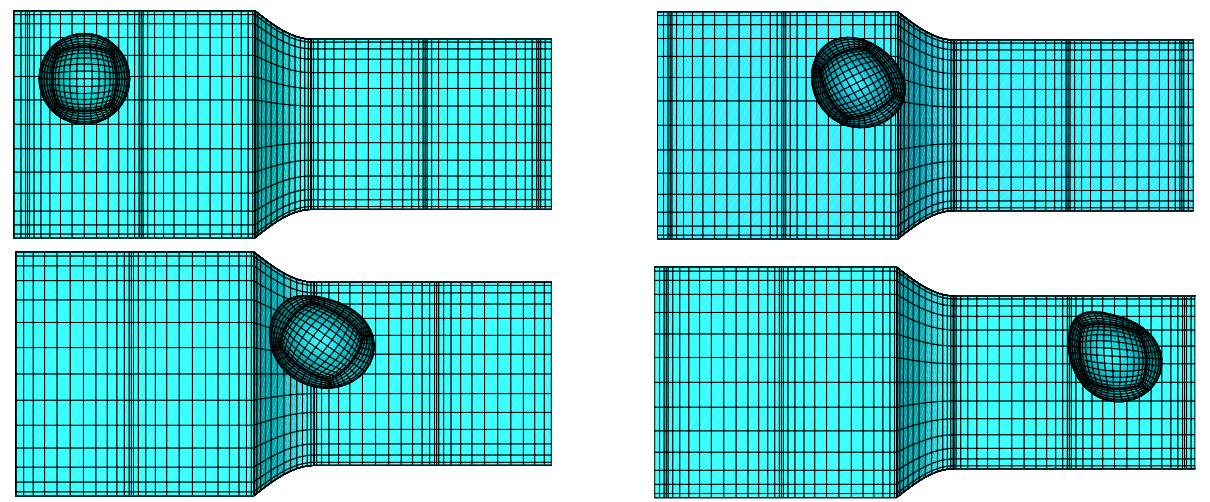

Figure 3. Lateral migration of a Skalak capsule with $\mathrm{Ca}=0.1, C=1, \lambda=1, a / \ell=0.8$ and initial position $z_{c}^{0} / \ell=0.8$ moving inside the constriction. Row-wise, the capsule centroid is $x_{c} / \ell=$ $-4,-1.71,0.103,2.76$.

To provide more details on the capsule's transient deformation at different initial positions, in Figure 4 we present the evolution of the capsule dimensions along the coordinate axes. In all cases, the capsule deforms significantly inside the constriction while the shape elongation is accompanied by a reduction of its width and height to accommodate the constant capsule volume. After the constriction, the capsule deformation is reduced. In this location, the capsule which was placed initially further away from the device centerline is more deformed owing to the stronger wall effects and thus migrates faster towards the device's centerline. Clearly, the proposed converging micro-capillary facilitates the capsule's migration towards the device centerline considerably. 

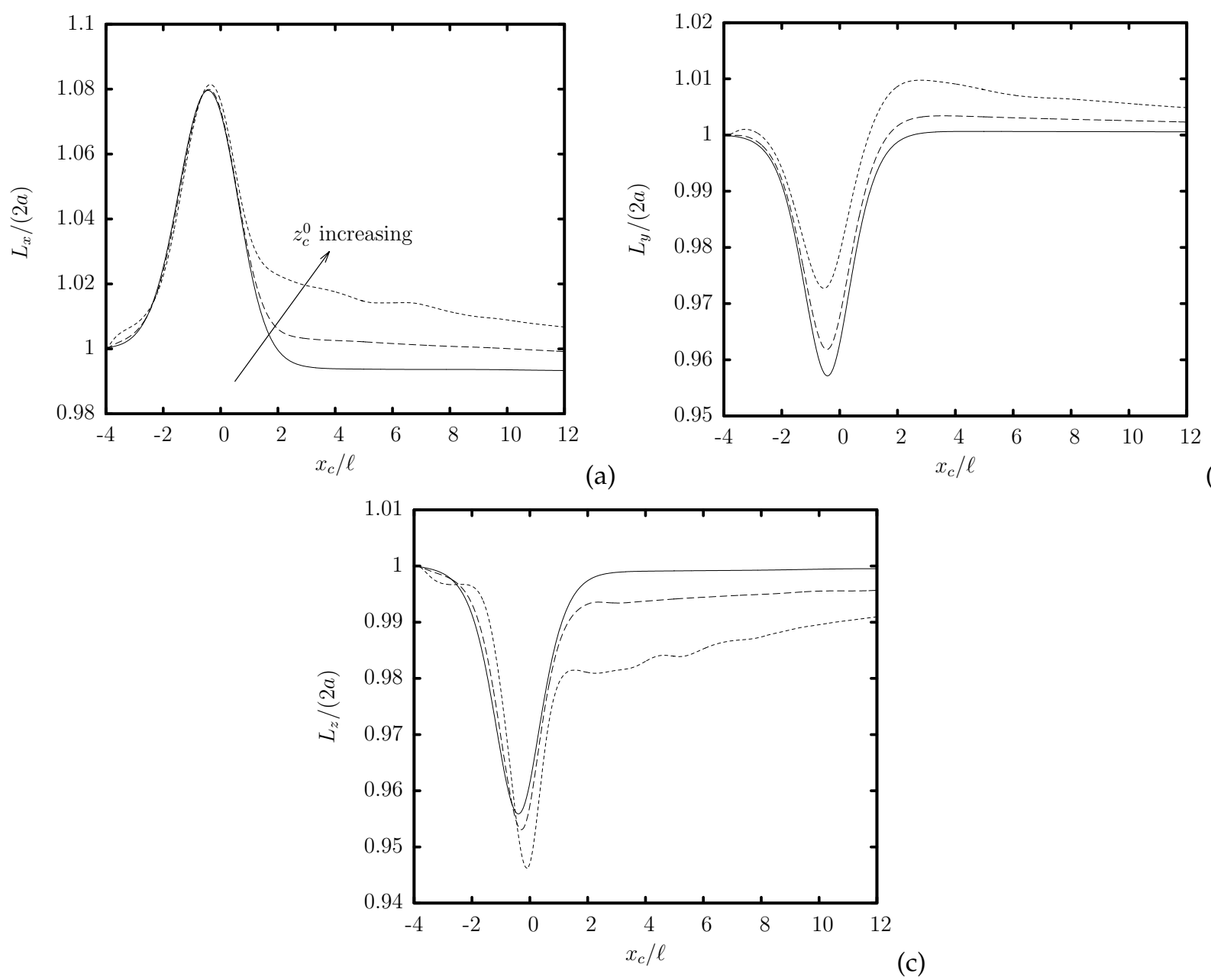

(b)

(c)

Figure 4. The capsule lengths as a function of the centroid $x_{c}$, for a Skalak capsule with $a / \ell=0.8, \mathrm{Ca}=0.1, C=1, \lambda=1$ and initial position $z_{c}^{0} / \ell=0.2,0.5,0.8$. (a) Length $L_{x},(\mathbf{b})$ width $L_{y}$ and (c) height $L_{z}$.

We investigate now the effects of the capsule size by considering the lateral migration of Skalak capsules with the same initial position $z_{c}^{0} / \ell=0.5$ and different sizes presented in Figures 5 and 6.
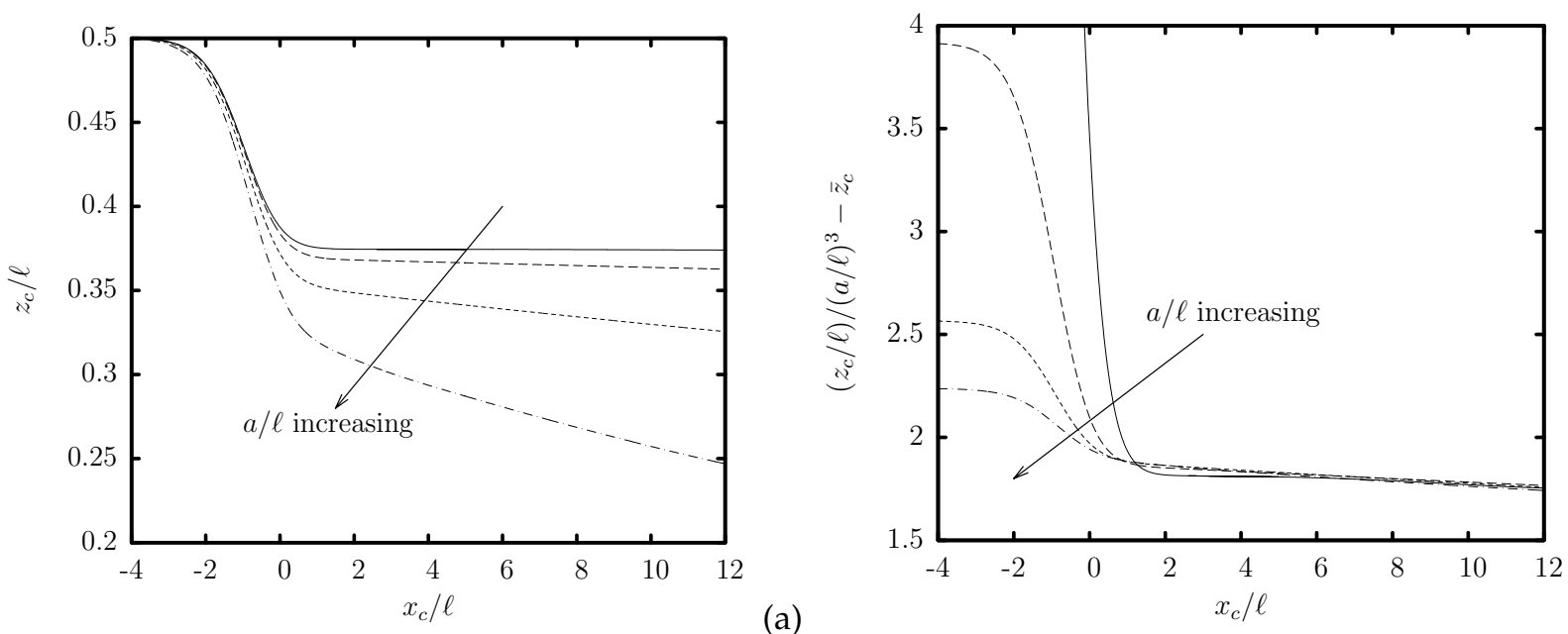

(b)

Figure 5. Capsule properties as a function of the centroid $x_{c}$, for a Skalak capsule with $\mathrm{Ca}=0.1, C=1, \lambda=1, z_{\mathcal{c}}^{0} / \ell=0.5$ and capsule sizes $a / \ell=0.2,0.4,0.6,0.8$ moving inside the constriction. Our results for $a / \ell=0.1,0.2$ are practically identical. (a) Capsule centroid $z_{c}$. (b) As in (a) but for the scaled centroid $\left(z_{c} / \ell\right) /(a / \ell)^{3}-\bar{z}_{c}$, where $\bar{z}_{c}$ is subtracted so that the curves have practically the same value at $x_{c} / \ell=12$. 
Our computations revealed that the smaller capsules with size $a / \ell=0.1,0.2$ have practically identical lateral migration. These small capsules at the flow rates studied in this work showed very small interfacial deformation, and thus they practically moved like spherical capsules; their path was quite close to the streamline of the single-phase flow passing though the initial position $z_{c}^{0} / \ell=0.5$ at the upstream channel. When the capsule size increased to $a / \ell=0.6,0.8$, the stronger hydrodynamic forces on the capsule surface owing to the higher flow blocking caused a significant cross-streamline migration inside and downstream the constriction, as shown in Figure 5a, along with a significant interfacial deformation inside the constriction. As seen in Figure 6, as the capsule size $a$ increased, the capsule length $L_{x}$ increased and its height $L_{z}$ decreased; i.e., the interfacial deformation increased as the capsule flowed through the constriction. Observe that under the same conditions, a larger capsule flowing in the constriction produced a higher flow blocking and thus increased hydrodynamic forces which result in higher interfacial deformation.
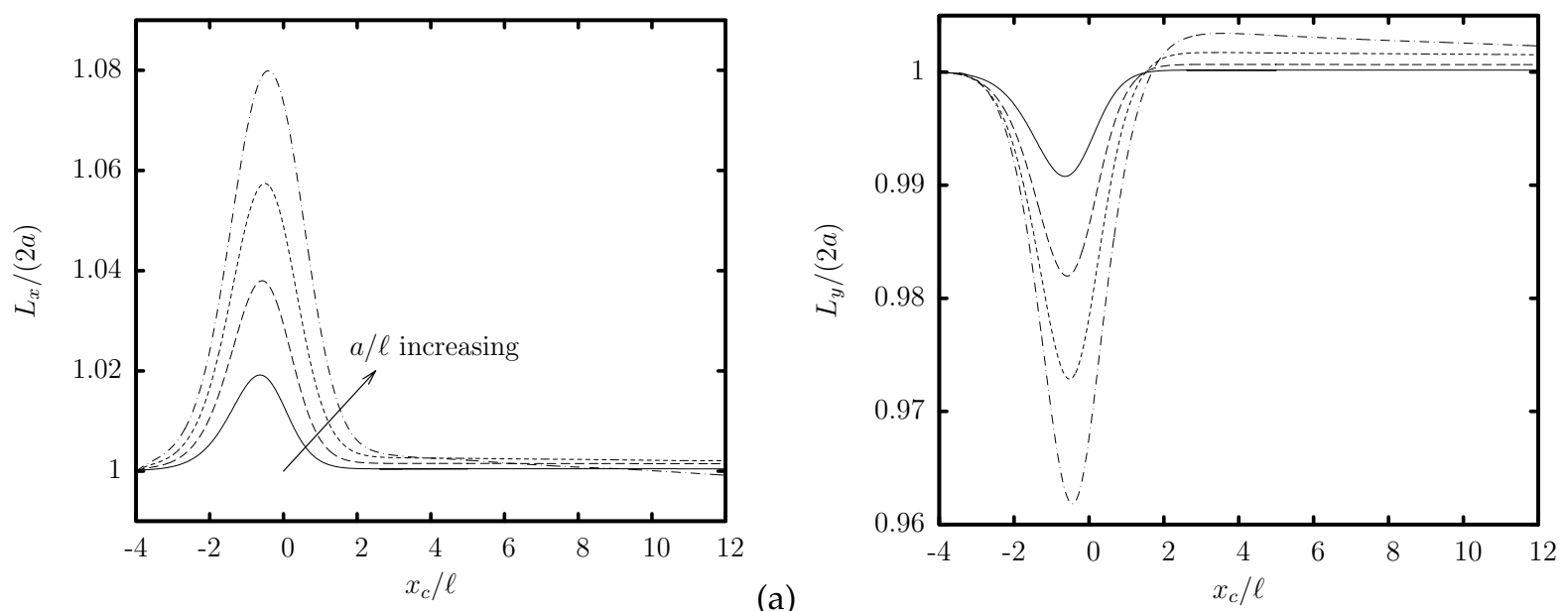

(a)

(b)

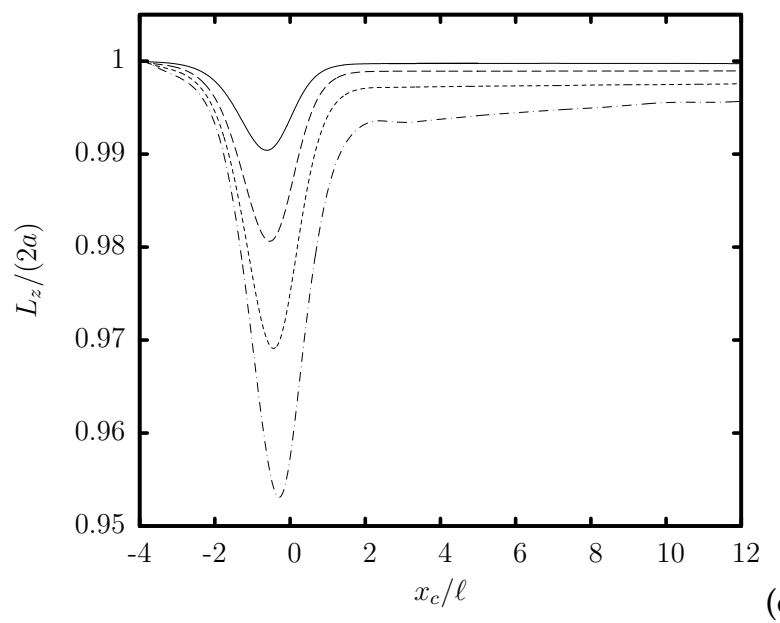

(c)

Figure 6. The capsule lengths as a function of the centroid $x_{c}$, for a Skalak capsule with $\mathrm{Ca}=0.1, \mathrm{C}=1, \lambda=1, z_{c}^{0} / \ell=0.5$ and capsule sizes $a / \ell=0.2,0.4,0.6,0.8$. (a) Length $L_{x},(\mathbf{b})$ width $L_{y}$ and (c) height $L_{z}$.

In essence, Figure 5a reveals that the curved streamlines of the single-phase flow inside the constriction facilitated the migration of large capsules towards the device centerline. In addition, in the downstream straight channel, the increased deformation of the larger capsules resulted in increased cross-streamline migration which scaled as $(a / \ell)^{3}$, as shown in Figure 5b:

$$
z_{c} \sim(a / \ell)^{3} x_{c} \quad \text { or } \quad U_{z} \sim(a / \ell)^{3} U_{x}
$$

We now turn our attention to the effects of the flow rate by considering the lateral migration of an elastic capsule with size $a / \ell=0.8$ and initial position $z_{c}^{0} / \ell=0.5$ at 
capillary numbers $\mathrm{Ca}=0.02,0.05,0.1$. As shown in Figure 7 , in these flow rates the capsule's transverse path is the same upstream and inside the constriction. Only after the constriction does the capsule with the higher capillary number migrate faster towards the device centerline.
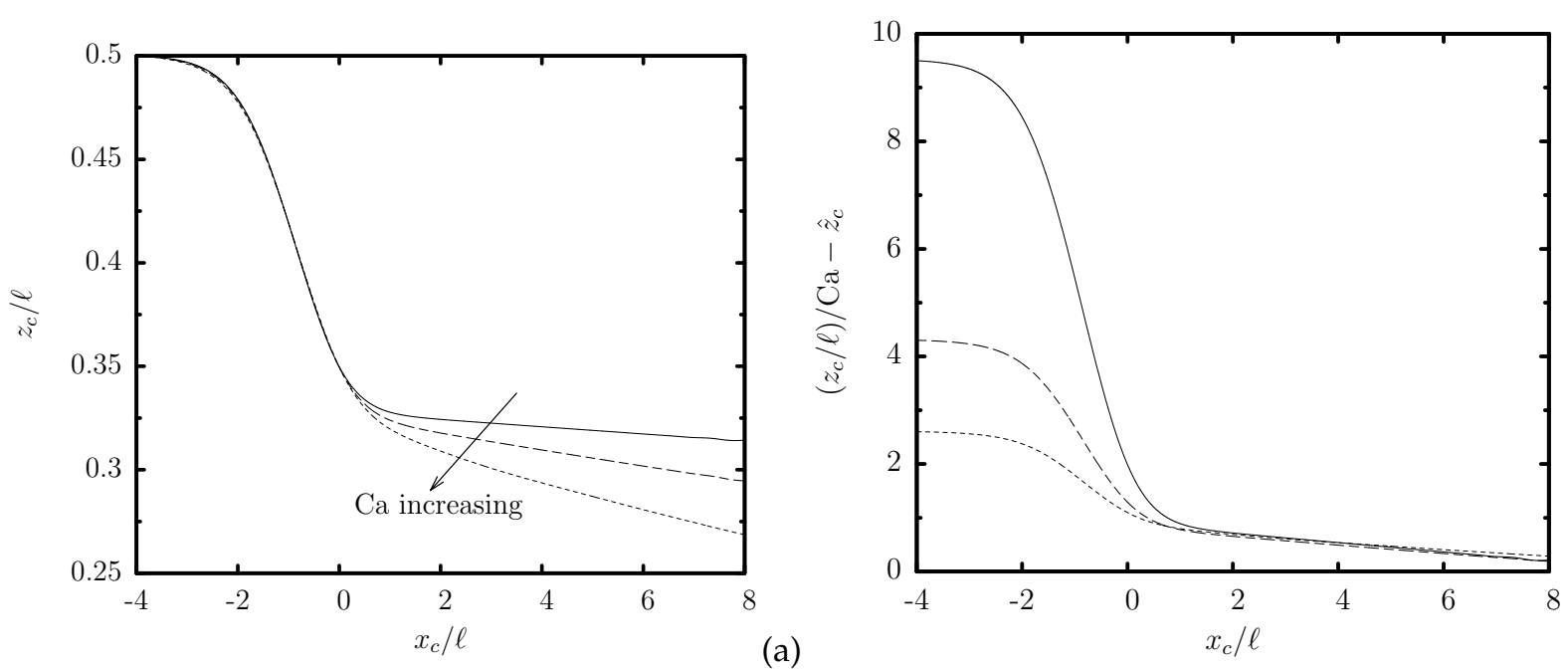

(b)

Figure 7. (a) The capsule centroid $z_{\mathcal{c}}$ as a function of the centroid $x_{\mathcal{c}}$, for a Skalak capsule with $a / \ell=0.8, C=1, \lambda=1$, $z_{c}^{0} / \ell=0.5$ and capillary numbers $\mathrm{Ca}=0.02,0.05,0.1$. (b) As in (a) but for the scaled centroid $\left(z_{c} / \ell\right) / \mathrm{Ca}-\hat{z}_{c}$, where $\hat{z}_{c}$ is subtracted so that the curves have practically the same value at $x_{c} / \ell=8$.

Therefore, the increased capsule deformation at the higher flow rates (presented in Figure 8), does not have any practical effects on the lateral migration over the curved streamlines inside the constriction. Thus, in this location, the lateral capsule migration is dominated by the strong hydrodynamic forces due to the large capsule size, which cause a significant cross-streamline migration for all the capillary numbers studied. However, in the downstream straight channel, the increased interfacial deformation at the higher flow rates results in increased lateral cross-streamline migration which is linearly proportionally to the capillary number, as shown in Figure $7 \mathrm{~b}$,

$$
z_{c} \sim \operatorname{Ca} x_{c} \quad \text { or } \quad U_{z} \sim \mathrm{Ca}_{x}
$$

It is of interest to note that both findings represented by Equations (3) and (4) are in agreement with the analytical predictions for the migration of a small droplet or capsule in an unbounded parabolic flow in the limit of small deformations [3,25].

We conclude our investigation by considering the effects of the viscosity ratio $\lambda$ and membrane hardness $C$ on the capsule lateral migration. Figure 9 presents the effects of viscosity ratio $\lambda$ and membrane hardness $C$ on the capsule lateral migration. In particular, both variables have practically no effects on the capsule lateral migration upstream and inside the constriction. Again, the significant cross-streamline migration occurring inside the constriction is dominated by the strong hydrodynamics forces of the surrounding flow due to the large capsule size. 
In contrast, after the constriction, the viscosity ratio and the membrane hardness do affect the capsule lateral migration via the interfacial deformation, as seen in Figure 9. In particular, the low-viscosity and equiviscous capsules (i.e., $\lambda=0.1,1$ ) exhibit practically the same interfacial deformation (not shown) and thus very similar lateral migration. The highviscosity capsule with $\lambda=5$ is affected by a much slower deformation rate, represented by the membrane time scale [23]

$$
\tau_{m} \sim(1+\lambda) \operatorname{Ca} \frac{a}{\ell} \tau_{f}
$$

(where $\tau_{f}=\ell / \mathcal{U}$ is the flow time scale), which reduces its lateral migration in the downstream square channel. In addition, as the membrane hardness $C$ increases, the increased area-dilatation resistance reduces the capsule deformation and its lateral migration, as shown in Figure 9b.
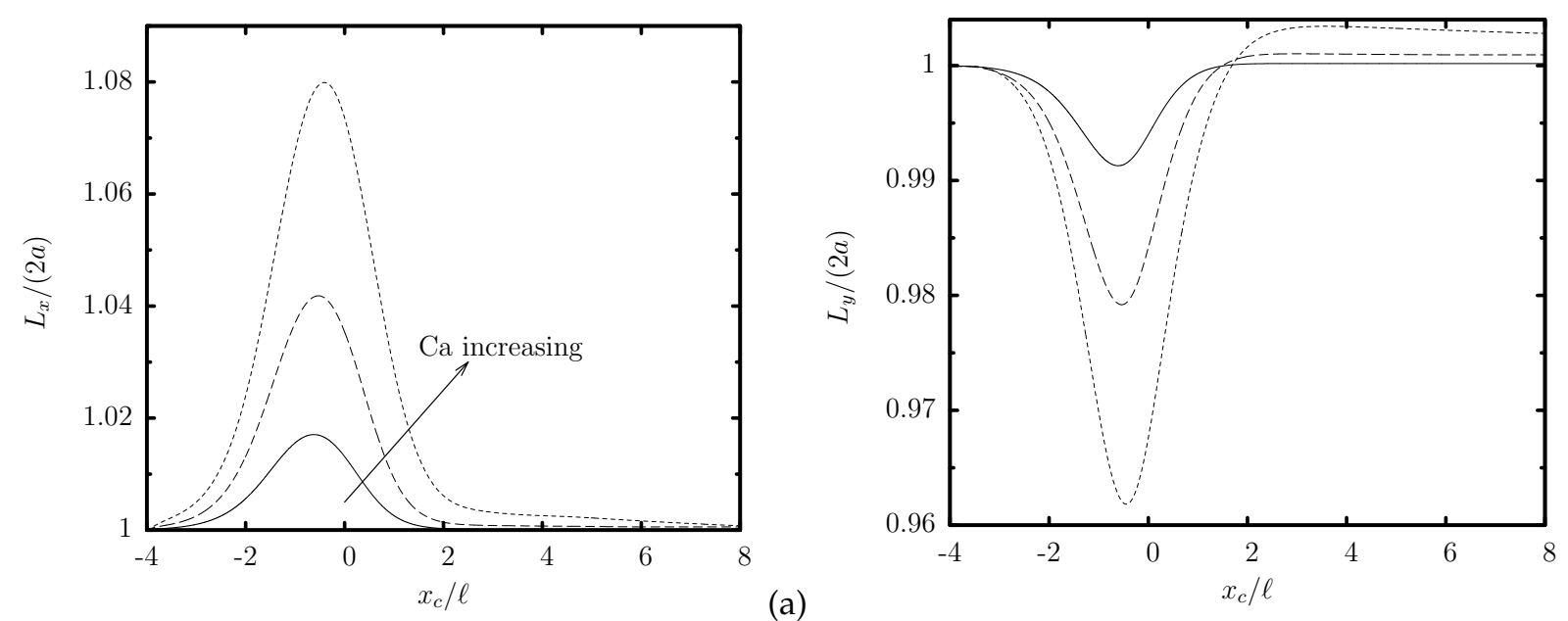

(b)

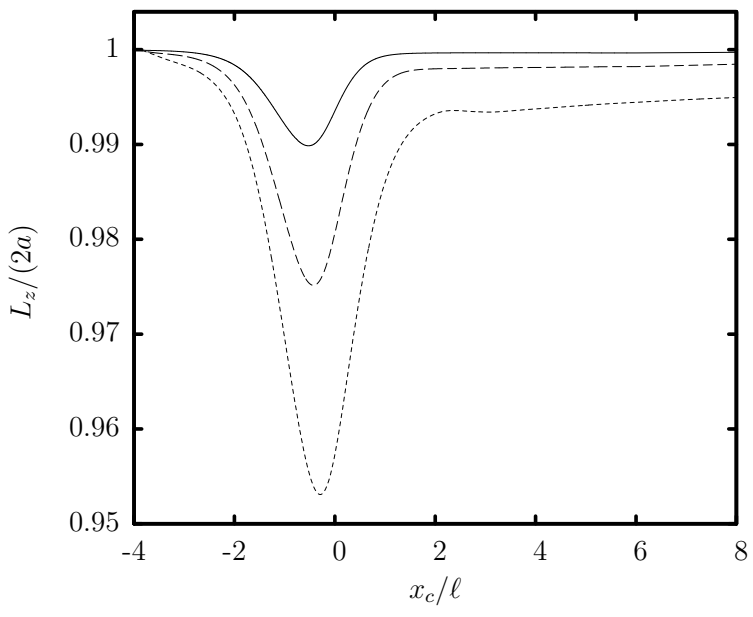

(c)

Figure 8. The capsule lengths as a function of the centroid $x_{c}$, for a Skalak capsule with $a / \ell=0.8, C=1, \lambda=1, z_{c}^{0} / \ell=0.5$ and capillary numbers $\mathrm{Ca}=0.02,0.05,0.1$. (a) Length $L_{x},(\mathbf{b})$ width $L_{y}$ and (c) height $L_{z}$. 

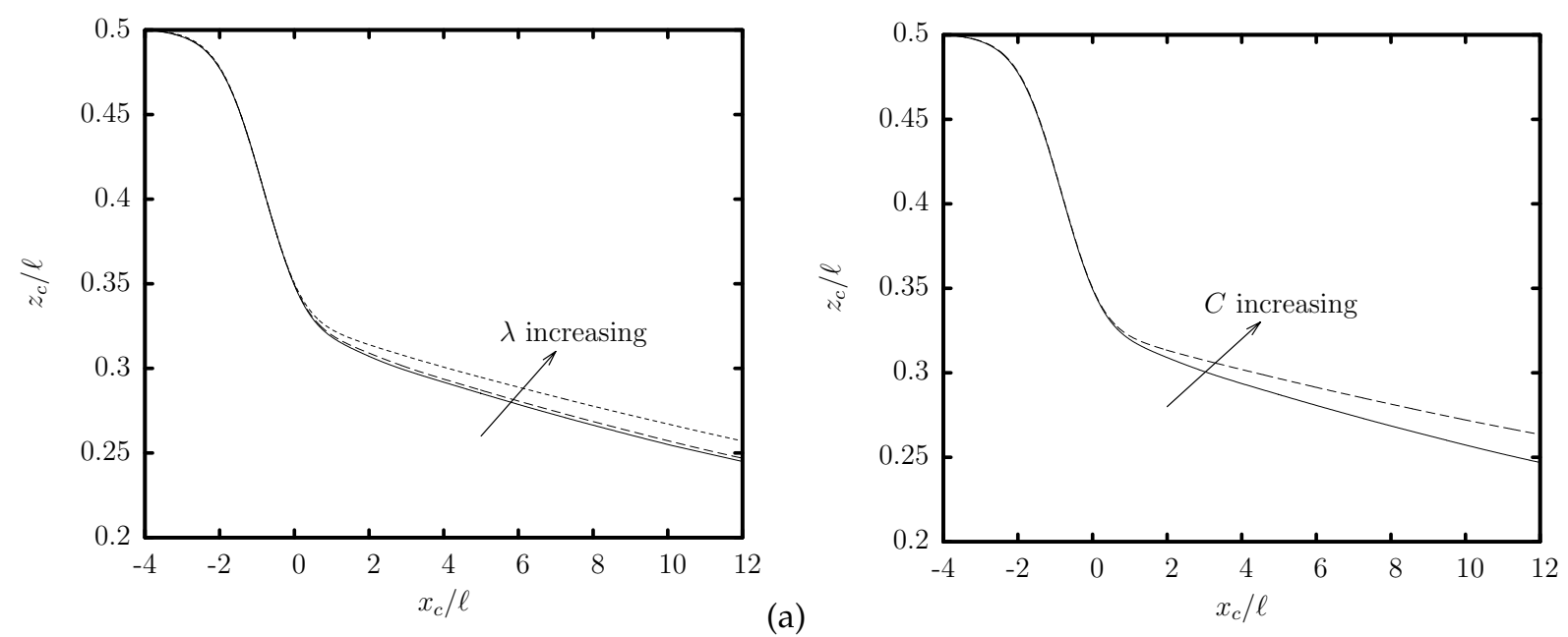

Figure 9. The capsule centroid $z_{c}$ as a function of the centroid $x_{c}$, for a Skalak capsule with $a / \ell=0.8, \mathrm{Ca}=0.1$ and $z_{c}^{0} / \ell=0.5$. (a) The viscosity ratio is $\lambda=0.1,1$ or 5 , and $C=1$. (b) The membrane stiffness is $C=1,2$ and $\lambda=1$.

\section{Conclusions}

In this work we have investigated the capsule motion in a converging micro-capillary as a medium to facilitate the cross-streamline migration towards a channel's centerline under Stokes flow conditions. Figure 7a clearly reveals that the increased capsule deformation at the higher flow rates does not have any practical effects on the lateral migration inside the constriction. Therefore, the significant cross-streamline migration over the curved streamlines inside the constriction is dominated by the strong hydrodynamic forces due to the capsule size. However, the interfacial deformation does facilitate the lateral migration over the straight streamlines of the downstream straight channel, as our results for varying capillary number, viscosity ratio and membrane hardness revealed. We emphasize that we have obtained similar behavior by utilizing a converging micro-capillary of circular cross-sections.

Thus our work highlights the different migration mechanisms occurring over curved and straight streamlines. We hope that our study motivates far more experiments of flowing elastic capsules in a variety of micro-geometries, involving curved streamlines.

Author Contributions: Conceptualization, P.D.; investigation, Y.W.; supervision, P.D.; writing, P.D. All authors have read and agreed to the published version of the manuscript.

Funding: This work was partially supported by the National Science Foundation (award 1335766).

Acknowledgments: The computations were performed on multiprocessor computers provided by the Extreme Science and Engineering Discovery Environment (XSEDE), and the Division of Information Techology at the University of Maryland.

Conflicts of Interest: The authors declare no conflict of interest.

\section{References}

1. Pozrikidis, C. Modeling and Simulation of Capsules and Biological Cells; CRC Press: Boca Raton, FL, USA, 2003.

2. Zhao, C.X. Multiphase flow microfluidics for the production of single or multiple emulsions for drug delivery. Adv. Drug Deliv. Rev. 2013, 65, 1420-1446. [CrossRef]

3. Chan, P.H.; Leal, L. The motion of a deformable drop in a second-order fluid. J. Fluid Mech. 1979, 92, 131-170. [CrossRef]

4. Coupier, G.; Farutin, A.; Minetti, C.; Podgorski, T.; Misbah, C. Shape diagram of vesicles in Poiseuille flow. Phys. Rev. Lett. 2012, 108, 178106. [CrossRef]

5. Magnaudet, J.; Takagi, S.; Legendre, D. Drag, deformation and lateral migration of a buoyant drop moving near a wall. J. Fluid Mech. 2003, 476, 115-157. [CrossRef]

6. Villone, M.M.; Maffettone, P.L. Dynamics, rheology, and applications of elastic deformable particle suspensions: A review. Rheol. Acta 2019, 58, 109-130. [CrossRef] 
7. Huh, D.; Gu, W.; Kamotani, Y.; Grotberg, J.B.; Takayama, S. Microfluidics for flow cytometric analysis of cells and particles. Physiol. Meas. 2005, 26, R73. [CrossRef]

8. Secomb, T.W.; Styp-Rekowska, B.; Pries, A.R. Two-dimensional simulation of red blood cell deformation and lateral migration in microvessels. Ann. Biomed. Eng. 2007, 35, 755-765. [CrossRef]

9. Singh, R.K.; Li, X.; Sarkar, K. Lateral migration of a capsule in plane shear near a wall. J. Fluid Mech. 2014, 739, 421. [CrossRef]

10. Hu, X.Q.; Sévénié, B.; Salsac, A.V.; Leclerc, E.; Barthès-Biesel, D. Characterizing the membrane properties of capsules flowing in a square-section microfluidic channel: Effects of the membrane constitutive law. Phys. Rev. E 2013, 87, 063008. [CrossRef] [PubMed]

11. Doddi, S.K.; Bagchi, P. Lateral migration of a capsule in a plane Poiseuille flow in a channel. Int. J. Multiph. Flow 2008, 34, 966-986. [CrossRef]

12. Nix, S.; Imai, Y.; Ishikawa, T. Lateral migration of a capsule in a parabolic flow. J. Biomech. 2016, 49, 2249-2254. [CrossRef]

13. Koolivand, A.; Dimitrakopoulos, P. Motion of an elastic capsule in a trapezoidal microchannel under Stokes flow conditions. Polymers 2020, 12, 1144. [CrossRef]

14. Utada, A.S.; Lorenceau, E.; Link, D.R.; Kaplan, P.D.; Stone, H.A.; Weitz, D. Monodisperse double emulsions generated from a microcapillary device. Science 2005, 308, 537-541. [CrossRef] [PubMed]

15. Duncanson, W.J.; Lin, T.; Abate, A.R.; Seiffert, S.; Shah, R.K.; Weitz, D.A. Microfluidic synthesis of advanced microparticles for encapsulation and controlled release. Lab Chip 2012, 12, 2135-2145. [CrossRef]

16. Wyss, H.M.; Franke, T.; Mele, E.; Weitz, D.A. Capillary micromechanics: Measuring the elasticity of microscopic soft objects. Soft Matter 2010, 6, 4550-4555. [CrossRef]

17. Dimitrakopoulos, P.; Kuriakose, S. Determining a membrane's shear modulus, independent of its area-dilatation modulus, via capsule flow in a converging micro-capillary. Soft Matter 2015, 11, 2782-2793. [CrossRef] [PubMed]

18. Skalak, R.; Tozeren, A.; Zarda, R.; Chien, S. Strain energy function of red blood cell membranes. Biophys. J. 1973, 13, 245-264. [CrossRef]

19. Barthès-Biesel, D.; Diaz, A.; Dhenin, E. Effect of constitutive laws for two-dimensional membranes on flow-induced capsule deformation. J. Fluid Mech. 2002, 460, 211. [CrossRef]

20. Dodson, W.; Dimitrakopoulos, P. Dynamics of strain-hardening and strain-softening capsules in strong planar extensional flows via an interfacial spectral boundary element algorithm for elastic membranes. J. Fluid Mech. 2009, 641, 263. [CrossRef]

21. Risso, F.; CollÉ-Paillot, F.; Zagzoule, M. Experimental investigation of a bioartificial capsule flowing in a narrow tube. J. Fluid Mech. 2006, 547, 149. [CrossRef]

22. Kuriakose, S.; Dimitrakopoulos, P. Motion of an elastic capsule in a square microfluidic channel. Phys. Rev. E 2011, 84, 011906. [CrossRef]

23. Park, S.Y.; Dimitrakopoulos, P. Transient dynamics of an elastic capsule in a microfluidic constriction. Soft Matter 2013, 9, 8844-8855. [CrossRef]

24. Koolivand, A.; Dimitrakopoulos, P. Deformation of an elastic capsule in a microfluidic T-junction: Settling shape and moduli determination. Microfluid. Nanofluidics 2017, 21, 89. [CrossRef]

25. Helmy, A.; Barthès-Biesel, D. Migration of a spherical capsule freely suspended in an unbounded parabolic flow. J. Mec. Theor. Appl. 1982, 1, 859-880. 